Review Article

\title{
An Overview on Herbal Cosmetics and Cosmeceuticals
}

\author{
Palle. Annie Jerusha ${ }^{1}$, Dr. K.V. Ratnamala*2 \\ ${ }^{1}$ M.Pharm (Pharmaceutics), RBVRR Women's College of Pharmacy, Affiliated to Osmania University, Hyderabad, Telangana, India. \\ ${ }^{2}$ Associate Professor (Department of Pharmaceutics), RBVRR Women's College of Pharmacy, Affiliated to Osmania University, \\ Hyderabad, Telangana, India. \\ *Corresponding author's E-mail: ratnakolapalli123@gmail.com
}

Received: 25-07-2021; Revised: 14-10-2021; Accepted: 24-10-2021; Published on: 15-11-2021. \begin{abstract}
Herbal cosmetics are formulated by using different cosmetic ingredients to form the base in which one or more herbal ingredients are wont to cure various skin ailment. The name suggests that herbal cosmetics are natural and free from all the harmful synthetic chemicals which otherwise may convince be toxic to the skin. Compared to other beauty products, natural cosmetics are safe to use. They are hypo-allergenic and tested and proven by dermatologists to be safe to use anytime, anywhere. Cosmeceuticals are cosmeticpharmaceutical hybrid products intended to enhance the health and wonder of the skin by providing a selected result, starting from acne-control and anti-wrinkle effects to sun protection. Cosmeceuticals have medicinal benefits which affect the biological functioning of skin depending upon the sort of functional ingredients they contain. These are cosmetic products that aren't just used for beautification but for different skin ailments. These products improve the functioning/texture of the skin by boosting collagen growth by eradicating harmful effects of free radicals, maintain keratin structure in good condition, and making the skin healthier. There are numerous herbs available naturally having different uses in cosmetic preparations for skin care, hair care, and as antioxidants. The current review highlights the importance of herbal cosmetics, the herbs used in them, and their advantages over their synthetic counterparts.
\end{abstract}

Keywords: Herbal cosmetics, Cosmeceuticals, Pharmaceutical hybrid products.

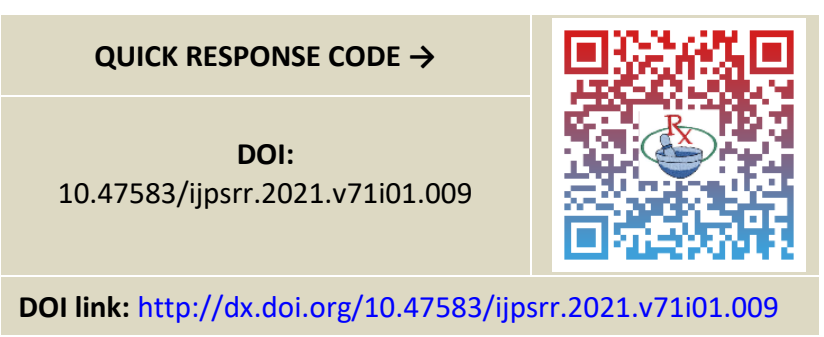

\section{INTRODUCTION}

T he development of beauty products and cosmetics is as old as civilization and the evolution of human beings. Every human being tries for their entities in physical and social facets of life which include the attraction of his/ her fiance. Hence it has become customary for human beings of all ages and climes to use cosmetics. A savage person whose worldly possessions are few, and whose daily means of sustenance is furnished by hunting and fishing also sticks feathers into his hair, paints his body with various designs and arranges his hairstyle in various fantastic forms as it gives him pleasure, raises him in the estimation of his fellow beings and has a magical significance to him. This attitude has been continuing in India from those ancient days and there was much-written evidence for the advancement of those beauty aids and cosmetics ${ }^{1}$.

\section{What are Cosmetics and Cosmeceuticals? ${ }^{2}$}

The word cosmetic was derived from the Greek word "kosmos tikos" meaning having the facility, arrange, skill in decorating. The origin of cosmetics forms endless narratives throughout the history of mankind as they developed from prehistoric times $3000 \mathrm{BC}$. The cosmetics, according to D \& C Act 1940 any article intended to be rubbed, poured, sprinkled, or sprayed on or introduced to or applied to any part of the human body for cleansing, beautifying, promoting the attractiveness which alters the appearance.

Cosmeceuticals are a combination of cosmetics and pharmaceuticals. Cosmeceuticals are cosmetic products that have biologically active ingredients purporting to possess medical or drug-like benefits. The "cosmeceutical" label applies only to those products which are applied topically such as creams, lotions, and ointments.

\section{What are Herbal Cosmetics? ${ }^{3}$}

Herbal cosmetics are defined as beauty products that helps in healing, smoothening, appearance, enhancing, and possessing desirable physiological activities for skin and skin conditioning properties with the help of herbal ingredients. These are the cosmetics that are prepared by using plant products having cosmetic actions. The base in which one or more herbal ingredients are used to provide defined cosmetic benefits and shall be called "Herbal cosmetics". The science of Ayurveda had utilized many herbs and floras to make cosmetics for beautification and protection from external effects. The natural content in the botanicals does not cause any side effects on the human body instead enriches the body with nutrients and useful minerals. 
Herbal cosmetics are comprised of floras like saffron(Kesar), ashwagandha, sandal (Chandan), and numerous increasingly that are expanded with healthy nutrients and all the various essential components. Herbal cosmetics like herbal conditioner, herbal soaps, herbal face wash, herbal shampoo, and lots of more are exceptionally acclaimed by the masses. The best thing of the herbal cosmetics is that it's purely made by the herbs and shrubs. The natural content within the herbs doesn't have any reactions on the physical body rather enhances the body with supplements and other helpful minerals. Herbs have a variety of functions such as food flavourings, cosmetics, and medicine in the forms of tea, tablet, capsule, tincture, cream, syrup, and liquid.

Herbal medicines include herbal materials, herbal preparations, and finished herbal products. Finished herbal products contain herbal preparations made up of one or more herbs. If one or more herbs are employed the term "mixture herbal product" also can be used. Herbs include crude material like leaves, flowers, fruits, seeds, stems, wood, bark, roots, rhizomes, or other plant parts which can be fragmented or powdered. Herbal cosmetics include herbal conditioner, herbal soaps, herbal face wash, herbal shampoo, and much more are exceptionally acclaimed by the masses. The best thing of the herbal cosmetics is that it's purely made by the herbs and shrubs. The natural content within the herbs doesn't have any reactions on the physical body rather enhances the body with supplements and other helpful minerals.

\section{Advantage of Herbal Cosmetics}

$\checkmark$ Herbal medicine features a long history of use and better patient tolerance and also as acceptance.

$\checkmark$ Medicinal plants have a renewable source, which is our only hope for sustainable supplies of cheaper medicines for the world's growing population.

$\checkmark$ The cultivation and processing of medicinal herbs and herbal products are environmentally friendly.

$\checkmark$ Prolong and apparently uneventful used for herbal medicines may offer testimony of their safety and efficacy.

$\checkmark$ Availability of medicinal plants isn't a haul especially in developing countries like India having rich agroclimatic, cultural and ethnic biodiversity.

$\checkmark$ They do not provoke allergies \& don't have negative side effects.

$\checkmark \quad$ They are easily incorporated within skin and hair.

$\checkmark$ With small quantities, they are very effective as compared to synthetic cosmetics.

$\checkmark$ Easily available in large variety \& quantity.

\section{Disadvantage of Herbal Cosmetics}

- Herbal drugs have slower effects as compare to allopathic dosage form. Also, it requires long term therapy.

- They are difficult to hide taste and odour.

- Manufacturing process are time consuming and complicated.

- No pharmacopoeia defines any specific procedure or ingredients to be used in any of herbal cosmetics.

- If an individual decides to take the herbal alternative to pharmaceuticals, he or she must be very patient.

- Herbal medicines are often self-administered. As a result, there is no dosage or warnings specified.

- When Herbal medicines are consumed with pharmaceutical drugs, the two can interact with each other resulting in injuries to health. It may be the case where a certain part of a plant may be edible and another part may be poisonous.

- Take rhubarb for example. The roots of rhubarb are used as a laxative and the stem is edible. However, its leaves are poisonous. An individual may not be able to identify a poisonous plant. This would put the individual at the risk of poisoning themselves or others.

\section{Present Status ${ }^{4-7}$}

Herbalists today, believe to assist people build their healthiness with the assistance of natural sources. Herbs are considered to be food instead of medicine because they're completely all-natural and pure, as nature intended. When herbs are taken, the body starts to urge cleansing, it gets purifying itself. Herbs don't produce instant cures, but rather offer how to place the body in proper tune with herbs. Herbs are utilized in the subsequent ways in cooking for flavouring the foods, as perfumes, as disinfectants, to guard us against germs, as medicines to heal once we are sick.

\section{Safe to Use}

Compared to other beauty products, natural cosmetics are safe to use and show effectiveness. They are hypoallergenic so they are tested and proven by dermatologists to be safe to use anytime, anywhere. Since they're made out of natural ingredients people don't need to worry about getting skin rashes or experience skin itchiness.

\section{Fits Your Budget}

Natural cosmetics aren't that expensive. In fact, a number of these products are cheaper than synthetic ones. They are offered at discounted prices and are sold for an inexpensive price during sales. Just got to survey enough to seem for nice deals. An estimate of WHO demonstrates about $80 \%$ of the world population depends on natural 
products for their health care, because of side effects inflicted and the rising cost of modern medicine. World Health Organization currently recommends and encourages traditional herbal cures in natural health care as these drugs are easily available at a low price and are comparatively safe.

\section{Not Tested on Animals}

Some cosmetics are initially tested on animals to ensure that they are safe and effective to use for humans. However, natural cosmetics don't need to be tested on animals. These natural preparations are tested by experts in laboratories using state-of-the-art equipment with no animals involved.

\section{No Side Effects}

Synthetic beauty products can irritate your skin and cause pimples. They might block your pores making your skin dry or oily. With natural cosmetics, one needn't worry about these. The natural ingredients used can have the assurance of no side effects one can apply them anytime, anywhere. As an example, herbal cosmetics are free from parabens that are the foremost widely used as preservatives in cosmetics and should penetrate the skin.

\section{Indian Extracts for Herbal Cosmetics}

Herbs play a vital role, especially in modern aims, when the damaging effects of food processing and overmedication have assumed alarming proportions. They are now being increasing cosmetics, foods and teas as well as alternative medicines. The growing interest in herbs may be a part of the movement towards change in life-styles.

\section{The Benefits of Herbs}

1. Enhance physical and mental well-being

2. Strengthen the immune system

3. Detoxification

4. Aid in sleeping, digestion

5. Increase stamina and reduce fatigue

6. Improves brain function and memory

7. Reduce nausea

8. Has anti-inflammatory effects

9. Helps fight infections

10. Helps reduce appetite

11. Have anti-cancer properties

12. Improves blood sugar levels

13. Help prevent allergies

14. Improve heart health.

\section{Herbs utilized in Cosmetics/Cosmeceuticals}

There are numerous herbs available naturally having different uses in cosmetic preparations for skincare, hair care, and oral care.

\section{Herbal Cosmetics Used For Skin ${ }^{8}$}

The Requirements for the Basic Skincare

\section{Cleansing agent}

It helps in removing the dust, dead cells and dirt that chokes the pores on the skin. Some of the common cleansers include vegetable oils like coconut, sesame and vegetable oil.

\section{Toners}

The toners help to tighten the skin and keep it from being exposed to several toxins that are floating within the air or other environmental pollutants. Some of the herbs which are used as toners are witch hazel, geranium, sage, lemon, essential oils.

\section{Moisturizing}

The moisturizer helps the skin to become soft and supple. Moisturizing shows a healthy glow and are less susceptible to aging. Some of the herbal moisturizers include vegetable glycerine, sorbitol, rose water, jojoba oil, Aloe vera and iris.

Whatever may be the type of skin, these three steps are performed to protect the skin from the constant effect of environment, stress and skin's natural process of cell degradation decay. Therefore, in order to help the skin look young and radiant.

- Exfoliation to remove dead skin cells.

- Epidermal stimulation for new cell growth.

- Antioxidant properties for cellular rejuvenation and repair.

- Improve capillary blood flow.

- Penetrating moisture and nutrients to replenish all layers of skin.

Skincare $^{9-11}$

Coconut oil

It is produced by crushing copra, the dried kernel, which contains $60-65 \%$ of the oil. Coconut oil contains a high number of glycerides of lower chain fatty acids. Coconut oil springs from the fruit or seed of the coconut tree coconut, Palmae. The freezing point of copra oil is 24 to $25^{\circ} \mathrm{C}$ (7576 F) and this is often used easily in liquid or solid forms and is usually utilized in cooking and baking. Coconut oil is superb as a skin moisturizer and softener.

\section{Sunflower oil}

It is the non-volatile oil extracted from sunflower seeds obtained from common sunflower composite. Sunflower oil consists of lecithin, tocopherols, carotenoids, and waxes. It has soothing properties and is taken into account as non-comedogenic. A simple yet cost-effective oil, tested for generations during a big variety of emulsions formulated for face and body products. 


\section{Jojoba oil}

It is a mixture of long-chain, linear liquid wax esters extracted from the seeds of the desert shrub Simmondsia chinensis family Simmondsiaceae. Jojoba oil is definitely refined to get rid of any odour, colour and it is oxidatively stable and is usually utilized in cosmetics as a moisturizer and as a carrier oil for exotic fragrances. Human sebum and jojoba oil are identical. Sebum protects and moisturizes the skin and hair but it strips away the chemicals, pollutants, sun, and aging process, leading to dry skin and hair. Jojoba oil replenishes skin and hair loss and restores them to their natural $\mathrm{pH}$ balance.

\section{Olive oil}

This is a fatty oil extracted from the fruits of olive, Oleaceae. The major components are triolein, tripalmitin, trilinolein, tristearate, monostearate, triarachidin, squalene, $\beta$-sitosterol and tocopherol. It is used in skin and hair as a conditioner in cosmetics like lotions, shampoos etc. It is a potent fatty acid that helps as a penetrating enhancer.

\section{Aloe vera}

Aloe vera is an herbal plant species belonging to the Liliaceae family that is found in naturally occurring populations, although closely related aloes do have a presence in northern Africa. It is an ingredient in many cosmetic products because it heals, moisturizes, and softens skin. Aloe vera contains amino acids like leucine, isoleucine, saponin glycosides that provide cleansing action, vitamins A, C, E, B, choline, B12 and supply antioxidant activity.

\section{Anti-Aging ${ }^{12}$}

\section{Rhodiola-rosea}

It's commonly referred to as golden root, rose root, common mullein, arctic root, king's crown, lignum rhodium, orpin rose. It is a plant in the Crassulaceae family that habitats in cold regions of the world. It grows mainly in dry sandy ground at high altitudes within the arctic areas of Europe and Asia, Traditional folk medicine used R. rosea to extend physical endurance, work productivity, longevity, resistance to high hypoxia, and to treat fatigue, depression, anaemia, impotence, gastrointestinal ailments, infections, and systema nervosum disorders. R. rosea is rich in phenolic compounds and known to have strong antioxidant properties.

\section{Carrot}

It is obtained from the plant wild carrot belonging to Apiaceae. It is a valuable herb since ages as it has richness in vitamin A alongside other essential vitamins. Carrot seed oil is employed as anti-aging, revitalizing and rejuvenating agent. The carrot gets its characteristic and bright orange colour from $\beta$-carotene and lesser amounts of $\alpha$-carotene and $\gamma$-carotene. $\alpha$ and $\beta$-carotenes are partly metabolized into vitamin $A$ in human beings.

\section{Gingko $^{14}$}

In China and Japan, the leaves and nuts of the Ginkgo ( $G$. biloba) tree are used for thousands of years to treat various medical conditions, including poor blood circulation, hypertension, poor memory, and depression, particularly among the elderly male impotence. In addition, it's gaining an identical reputation as an antioxidant and anti-inflammatory agent. Ginkgo biloba belongs to Ginkgoaceae which grows to an enormous $\operatorname{size}^{25}$. The G. biloba extract E. Gb 761, prepared from the tree leaves, and it is a natural mixture containing flavone glycosides (33\%), mostly quercetin and kaempferol derivatives, and terpenes (6\%), which has exhibited the capacity to isolate from the leaves of $\mathrm{L}$. inermis has shown significant antifungal and antibiotic effect .

\section{Neem}

Neem or Margosa is a botanical relative of mahogany. It belongs to the family Meliaceae. The Latin name of NeemAzadirachta indica is derived from the Persian. Azad=Free, dirakht=Tree, $\mathrm{i}-\mathrm{Hind}=\mathrm{of}$ Indian Origin. The common treatment for the dandruff is Neem because it produces antifungal, antibacterial, pain-relieving, and anticompounds that might treat dandruff.

\section{Skin Protection ${ }^{15-17}$}

\section{Green tea}

The tea plant (Camellia sinensis) has been cultivated in Asia from many years. The four major polyphenolic catechins present in tea leaves are (2)-epicatechin (EC), EGC, (2)-EC3-gallate, and EGCG which is that of the most abundant. It was found that green tea extracts or an individual green tea polyphenol (GTPP), especially epigallocatechin (EGC)3-gallate (EGCG), inhibited two-stage chemical carcinogenesis (e.g., induced by 7,12-dimethylbenz(a)anthracene [DMBA] and 12-Otetradecanoylphorbol 13-acetate [TPA]), and photo carcinogenesis (induced by UVB).

\section{Calendula}

Calendula officinalis have a remarkable antioxidant activity, anti- inflammatory activity and wound healing activity. A previous study demonstrated that the essential oil of Calendula consists of important components like $\alpha$ thujene, $\alpha$-pinene, 1,8-Cineole, dihydrotagetone and Tmuurolol.

\section{Turmeric}

It is a deep yellow-to-orange powder that reduce the number of ultraviolet B (UVB)-induced sunburn cells in mice. Turmeric contains good number of phytochemicals including, demethoxycurcumin, bisdemethoxycurcumin, zingiberene, curcumol, curcumenol, eugenol, tetrahydrocurcumin, triethylcurcumin, curcumin, turmerin, turmerones, and turmeronols. Curcuministhephytochemical that provides a yellow color to turmeric and is now recognized as being liable for most of the therapeutic effects. Turmeric uses include 
antiseptic, analgesic, anti-inflammatory, antioxidant, antimalarial, insect- repellant, and other activities associated to turmeric.

\section{Types of Herbal Hair Care Products ${ }^{18}$}

\section{Hair Oil}

The hair oils are used for dressings and nourishing the hair follicles. These preparations are generally used to increase the growth of hair and to make them healthy.

Hair oil should have following properties:

$\checkmark$ They should give luster (soft glow) to the hair.

$\checkmark$ Retain them soft and flowing.

$\checkmark \quad$ Invigorate their growth.

$\checkmark \quad$ Keep the brain cool.

$\checkmark$ Should not be sticky.

\section{Hair Lotion}

Hair lotion has a stimulating effect on the hair follicles. They are generally perfumed with oil of rosemary as it possess a good stimulating property.

\section{Hair Colourants}

These are preparations which are used for the colouring of the hairs. They enhance the attractiveness of grey hair. They are applied externally on the hair with help of a brush.

\section{Hair Shampoo}

Shampoo is preparation of surfactant in suitable form of liquid, solid or powder- when used under the condition specified will remove surface grease, dirt, and skin debris from the hair shaft and scalp.

\section{Qualities of an Ideal Hair Care Product}

It should:

$\checkmark$ Protect the hair cuticle.

$\checkmark$ Cleanse without stripping natural oils.

$\checkmark$ Replace lost protein, moisture, and nutrients.

$\checkmark$ Condition without weighing down the hair.

$\checkmark \quad$ Even without porosity and prevent moisture loss.

$\checkmark$ Smooth abraded cuticle scales.

$\checkmark$ Prevent intense drying from environment.

\section{Dandruff Treatment ${ }^{19}$}

Ayurveda has numerous natural medications wherein the most common herbs include Neem, Kapoor (naphthalene), and Henna, Hirda, Baheda, and Amalaki, Magic nut, Bringaraj, Rosary Pea, Sweet Flag, Cashmere tree and Mandor.

\section{Henna}

Henna comes from the plant Lawsonia inermis, Lythraceae which contain a dye molecule called Lawsone, which when processed produces Henna powder. Besides lawsone there are other constituents present are acid, glucose, mannitol, fats, resin (2\%), mucilage and traces of an alkaloid. Leaves yield henna tannin and a vegetable oil green resin, soluble in ether and alcohol. Lawsone edible fruit which has highly praised both for its high vitamin $\mathrm{C}$ content and for the valuable oil, which is extracted from its seeds and pulp and used as a treatment for hair and scalp problems. It is used in eye syndromes, hair loss, and children illness etc.

\section{Shikakai}

Acacia concinna linn. (Leguminosae) it is a medicinal plant that grows in tropical rainforests of southern Asia. The fruits of this plant are used for laundry hair, for improving hair growth, as an expectorant, emetic, and purgative. The powder of Acacia concinna linn shows the existence of saponins, alkaloids, sugar, tannin, flavonoids, anthraquinone glycosides.

\section{Herbal Ingredients in Oral Care ${ }^{20}$}

In many traditional cultures, there are no plastic-bristle brushes rather the utilization of herbal "chewing sticks" are common. Chewing sticks are taken from plants, shrubs or trees with high anti-microbial activity. The ends of selected sticks are shredded and that they are used to massage the gums and "floss" the teeth. Young twigs of any variety of Neem, oaks, willows have served for chewing sticks, and are still available today Some of the important herbs and their use in dentistry and oral care as follows:

\section{Clove Oil (Syzygium aromaticum)}

Clove oil has long been applied directly to the gums to ease toothache. There is evidence that the eugenol in clove oil is effective at fighting several known oral bacteria. Medicines containing eugenol are widely used in dentistry. Some research suggests that clove gel may reduce the pain of needle insertion in dentistry. It has got excellent analgesic and antiseptic properties which inhibit the growth of all disease-causing bacteria. Helps in treating the bleeding gums.

\section{Aloe-Vera (Ghritkumari)}

Aloe-vera has anti-inflammatory and antibacterial properties. The plant is also rich in antioxidants. For this reason, people use aloe vera to treat and relieve inflammation related to gum disease and other oral issues. Aloe vera also contains powerful antioxidants that fight free radicals that cause cell damage. This aloe vera may help the following conditions:

- gingivitis

- gum infections or periodontitis

- $\quad$ other irritation in the mouth

\section{Tea Tree Oil (Melaleuca alternifolia) ${ }^{21}$}

Tea tree oil is a popular component of various at-home dental remedies, said to help control plaque, freshen 
breath, ease tooth pain, and treat periodontal disease. It has antiseptic, antifungal properties. And used in the treatment of throat irritation.

\section{Garlic (Allium sativum)}

One of the most well-known compounds in garlic is allicin, which has antibacterial property that can help kill some of the bacteria associated with toothaches. Allicin is found in fresh garlic after it is crushed or cut. It has antibacterial activity depends on allicin produced by enzymatic activity of alliinase. Used to inhibit the growth of Streptococcus mutants, and therefore can be used as an effective remedy in the prevention of dental caries.

\section{Neem (Azadirachta indica)}

Oral hygiene is the practice of keeping the mouth and teeth clean to prevent dental problems most commonly in dental cavities, gingivitis, and bad breath. Used in preventing and healing gum diseases and other dental problems such as bleeding and plaque.

\section{Thyme (Thymus vulgaris)}

Thyme oil exhibits antibacterial activity and has been useful in dental practice. A component of thyme, known as thymol, appears to inhibit growth of oral decay. It is mainly composed of volatile oils namely phenol, thymol and carvacrol. Used in toothpaste.

\section{Turmeric (Curcuma longa Linn.)}

Turmeric is a completely natural product, which makes it a desirable alternative to less natural, commercial teeth whiteners. Possible benefits you can get from using turmeric for teeth whitening are teeth whitening, reduced risk of gum pain and inflammation, reduced risk of gum disease. And it is an antiseptic, antibacterial, antiinflammatory and painkiller.

\section{Meswak (Salvadora persica)}

It is a derivative from Arak tree. The use of Miswak appeared to be more effective than tooth brushing for removing the plaque from the embrasures thus, enhancing interproximal oral health.

\section{Herbal Tooth Preparations ${ }^{22}$}

To prevent and control the teeth disorders, which include herbal tooth paste, herbal tooth powder, dentifrices and mouthwash.

\section{Dentifrices}

The basic requirements of a dentifrice are-

1. To remove food debris, plaque and stain.

2. It should leave the mouth with a fresh, clean sensation.

3. It should be harmless, pleasant and convenient to use.

\section{Tooth paste}

To remove adherent soil matter from a hard surface with little damage. It is used to promote oral hygiene and it is an abrasive that aids in removing dental plaque and food from the teeth, help to prevent tooth decay and gum disease.

\section{Mouth wash}

Mouthwash or mouth rinse may be a product want to enhance oral hygiene. It is an antiseptic to kill bacteria that can live between your teeth and on your tongue. Some people use mouthwash to fight against bad breath, while others use it to undertake to stop cavity.

\section{Herbal Toothpastes}

1. Divya Dant Kanti Tooth Paste:

2. Vajradanti Extract

3. Pilu Extract

4. Babool Extract

5. Akarkara Extract (Anacyclus Pyrethrum)

6. Majuphal Extract (Quercus infectoria)

7. Kapur Extract (Cinnamomum camphora)

8. Vidang Extract

9. Nimba or Neem Extract

10. Lavang Extract

\section{Hiora Mouthwash}

Key Ingredients:

1. Pilu (Salvadora persica)

2. Bibhitaka (Terminalia bellerica)

3. Nagavalli (Piper betle)

4. Gandhapura taila (Gaultheria fragrantissima)

5. Peppermint satva (Mentha sp)

6. Yavani satva (Trachyspermum ammi)

\section{Essential Oils ${ }^{23}$}

$>$ Essential oils (EOs) are distilled liquids extracted from flowers, leaves, bark, stems, roots, shrubs and trees.

$>$ The effectiveness of EOs lies in their exceptional ability to penetrate human tissue and provide therapeutic benefits.

$>$ EOs contains varying numbers of tiny aromatic molecules that rapidly absorb into skin and travel through the body via blood capillaries and the circulatory system.

$>$ When used in herbal dental care, EOs is absorbed deep into gum tissue where their potent 
antibacterial properties provide valuable benefits in the treatment of gum disease.

\section{Red Thyme}

The oil from this herb has been used extensively in medicine as a strong antiseptic and disinfectant. Additionally, it's antibacterial, antifungal, antiinflammatory, antimicrobial, and stimulating properties to assist us treat oral inflammation and infection, making it a strong additive to herbal dental products.

\section{Cinnamon Bark}

The high aldehyde content of this oil makes it a robust antimicrobial and antiseptic. High in tannins, cinnamon bark is an astringent but provides valuable benefits. Astringent strengthens oral tissue, reduce surface inflammation and irritation, and makes a protective barrier from infection. Cinnamon oil is widely known as a painkiller and has long been want to soothe toothaches.

\section{Peppermint $^{24}$}

Oil from this plant provides antibacterial, antiinflammatory, antifungal, antimicrobial, antiseptic, sedative and stimulant properties. While peppermint flavouring is one of the foremost common additives utilized in commercial toothpaste and mouthwash, the bulk of those products don't contain the volatile oil itself.

\section{Lavender}

It is widely used for its skin healing properties, the oil from this highly aromatic flower offers antibacterial, antiinflammatory, antimicrobial, antiseptic and stimulant properties in natural dental remedies, making it a strong ally for good oral health. Its pleasant aroma is additionally a strong sedative which helps to calm the systema nervosum and reduce stress.

\section{Rose oil}

Roses are widely referred to as the world's favourite flower in part due to their vast diversity in plant habitat and floral from the underground stems of the tropical perennial herb turmeric of the Zingiberaceae. Turmeric contains a good range of phytochemicals including, dimethoxy curcumin, bisdemethoxy curcumin, zingiberene, curcumol, curcumenol, eugenol, tetrahydro curcumin, triethyl curcumin, turmerin, turmerones, and turmeronols. Curcumin is the phytochemical that provides a yellow colour to turmeric and is now recognized as being liable for most of the therapeutic effects. Turmeric uses include antiseptic, analgesic, anti-inflammatory, antioxidant, antimalarial, insect- repellant and other activities associated to turmeric.

\section{Eucalyptus oil ${ }^{25}$}

There are around 700 different species of Eucalyptus in the world, of which at least 500 species produce a type of essential oil. Oil produced by steam distillation from the leaves of Eucalyptus species (E. cinerea F. Muell., E. baueriana F. Muell., E. smithii R. T. Baker, E. bridgesiana R.
T. Baker, E. microtheca F. Muell., E. foecunda Schau, E. pulverulenta Sims, E. propinqua Deane and Maiden, E. erythrocorys F. Muell.) etc. They are widely used in the pharmaceutical preparations like liniments, inhalants, cough syrups, ointments, toothpaste and also as pharmaceutical flavours. The European Pharmacopoeia monograph for essential oil sports a chromatographic profile i.e., 1,8-cineole (eucalyptol; not less than70\%), limonene (4-12\%), $\alpha$-pinene (2-8\%), $\alpha$ - phellandrene (less than $1.5 \%), \beta$-pinene (less than $0.5 \%$ ), camphor (less than $0.1 \%)$.

\section{Anti-Oxidants ${ }^{26}$}

\section{Tamarind}

Tamarind or Tamarindus indica of the Fabaceae consists of amino acids, fatty acids and minerals of tamarind plant parts. The most distinguished characteristic of tamarind is its sweet acidic taste thanks to hydroxy acid. Besides being an upscale source of sugars, tamarind fruit is additionally a superb source of B-complex vitamin and contain minerals, exhibit high antioxidant capacity that appear to be associated with a high phenolic content, and thus are often a crucial food source.

\section{Vitaminc $^{27}$}

VitaminC is important for the hydroxylation of proline, procollagen, and lysine. Vitamin $\mathrm{C}$ improves the damage caused by photos. Vitamin $C$ has been used effectively to stimulate collagen repair, thus removing a number of the consequences of photo-aging on skin.

\section{Vitamin $E^{28}$}

(Alpha-tocopherol) is the major lipophilic antioxidant in plasma membranes and tissues. The term vitamin $E$ collectively refers to 30 present molecules (4 tocopherols and 4 tocotrienols), all of which exhibit vitamin E activity. Its important role is generally considered to be the arrest of chain propagation and lipid peroxidation by scavenging lipid peroxyl radicals, hence protecting the cell membrane from destruction.

\section{CONCLUSION}

In India more than $70 \%$ of the populations use herbal cosmetics for their health care. At present time herbal cosmetic has been marked up in personal care system and there is a great requirement for the herbal cosmetics in daily life. Healthy teeth, shiny hair and glowing skin are significant for the good looking of the human body. The demand of herbal cosmetics is rapidly expanding. The advantages of herbal cosmetics are lower cost, side effects free, environmentally friendly, safe to use etc. Also has a great future ahead as compared to the synthetic cosmetics. Proper regulation of these herbs and standardization will lead to tremendous and significant growth in herbal cosmetics field. 


\section{REFERENCES}

1. Gediya SK, Blessy $M$ and Jain HN, Herbal plants: Used as cosmetics, 2011; 1: 24-32.

2. Athar M, Taxonomic perspective of plant species yielding vegetable oils used in cosmetics and skin care products, African Journal of Biotechnology, 2005; 4: 36-44.

3. Rabasco AAM, Gonzalez RML, Lipids in pharmaceutical and cosmetic preparations, Grasas y Aceites, 2000; 51: 74-96

4. Basmatekar G, A valuable multifunctional cosmetic ingredient, Int J Med Aroma Plants, 2011; 1: 338-341.

5. Furmanowa $M$, Rhodiola rosea in vitro culture: phytochemical analysis and antioxidant action., Acta Societis Botanicorum Poloniae, 1998; 67: 69-73

6. Strube $\mathrm{M}$, Dragsted OL, Naturally occurring Antitumourigens. Iv. Carotenoids except $\beta$-carotene, 1999.

7. Jain A, Dubey S, Gupta A, Kannojia P, Tomar V, Potential of herbs as cosmeceuticals, IJRAP, 2010; 1: 71-77.

8. Dixit SN, Srivastava HS, Tripathi RD, Lawsone, The antifungal antibiotic from leaves of Lawsonia inermis and some aspects of its mode of action. Indian phytopathol, 1980; 31: 131-133.

9. Anand N, Aquicio JM, Anand A, Antifungal properties of Neem (Azadirachta indica) leaves extract to treat Hair Dandruff. E-International Scientific Research Journal, 2010; 2: 244-252.

10. Adhami VM, Mukhtar H, Ahmad N, Farrukh A, Yukihiko H, Tea polyphenols as cancer chemopreventive agents, $T$ cell Biochem suppl, 1995; 22: 169-180.

11. Katiyar SK, Elmets CA, Green tea polyphenols skin protection and antioxidant (Review). Int J oncol, 2001; 18: 1307-1313.

12. Mukhtar H, Katiyar SK, Agarwal R, Green tea and skin anticarcinogenic effects. J invests Dermatol, 1994; 102: 37.

13. Okoh OO, Sadimenko AP, Asekeen OT, Afolayan AJ, The effects of Drying on the chemical components of Essential oils of Caledula officinalis L. African J Biotechnol, 2008; 7: 1500-1502.

14. Fujiyama YF, Effects of sesamin and curcumin on delta 5desaturation and chain elongation of polyunsaturated fatty acid meabolism in primary cultured fatty acid metabolism in primary cultured rat hepatocytes, J Nutr sci vitaminol (Tokyo), 1992; 38: 353-363.

15. Chaudhari KR, Turmeric, haldi or haridra, in eye diseases. Antiseptic, 1950; 47: 67-68.
16. Nadkarni KM, Curcuma longa in India material, Popular Prakashan, 1976; 414-418.

17. Saikia AP, Ryakala VK, Sharma P, Goswami P, Bora U, Ethnobotany of medicinal plants used by Assamese people for various skin ailments and cosmetics, J Ethnopharmacol, 2006; 149-157.

18. Gediya SK, Mistry RB, Patel UK, Blessy M, Jain HN, Herbal plants : used as cosmetics, J Nat Prod Plant Resour, 2011; 1: 24-32.

19. Pandey S, Meshya N, Viral D, Herbs play an important role in the field of cosmetics, International Journal of Pharm Tech Research, 2010; 2: 632-639.

20. Khanpara K, Renuka V, Shukla J, Harsha CR, A Detailed Investigation of shikakai (Acacia concinna Linn) fruit. Journal of Current Pharmaceutical Research, 2012; 9: 0610.

21. Haghighi M, Tehranifar A, Nikbakht A, Kafi M, Research and current profile of Iranian production of Damask Rose (Rosa Damascena Mill). International society of Horticulture Science, 2008; 769: 449-455

22. Sefidkon F, Assareh MH, Abravesh Z, Barazandeh MM, Chemical composition of the Essential oils of four cultivated Eucalyptus species in Iran as medicinal plants [E. microtheca, E. spatbulata, E. largiflorens and E. torquata]. Iranian Journal of Pharmaceutical Research, 2007; 6: 135140.

23. Rao Diwan PV, Herbal formulation useful as therapeutic and cosmetic applications for the treatment of general skin disorders, 2001; US6200570 B1: March 13.

24. Marini JL, Cosmetic herbal compositions. EP1825845 A1, August 29th; 2007

25. Mengoli F, Herbal cosmetic compsns-contg. Herbs in mixt of surfactants, essential oils, etc., April 1 ${ }^{\text {st}} 1993$; DE4133085 A1.

26. Neelakantan K, Dry herbal, cleaning compositions, April $14^{\text {th }}$ 1999; EP0908171 A1.

27. Bonte F, Dumas M, Maybeck A, Cosmetic or dermatologic composition containing at least one saponine of the ginsenoside type, and its applications particularly to hair care, March 31 $1^{\text {st }}, 1994$; W01994006402 A2.

28. Kadam VS, Chintale AG, Deshmukh KP, Nalwad DN, Cosmeceuticals an emerging concept: A comprehensive Review. International journal of research in pharmacy and chemistry, 2013; 3: 308-316.

Source of Support: The author(s) received no financial support for the research, authorship, and/or publication of this article.

Conflict of Interest: The author(s) declared no potential conflicts of interest with respect to the research, authorship, and/or publication of this article.

For any question relates to this article, please reach us at: editor@globalresearchonline.net New manuscripts for publication can be submitted at: submit@globalresearchonline.net and submit_ijpsrr@rediffmail.com 